\title{
Highly Flexible, Electrically Driven, Top-Emitting, Quantum Dot Light- Emitting Stickers
}

\author{
Xuyong Yang, ${ }^{\dagger}$ Evren Mutlugun, ${ }^{t, \neq, \|}$ Cuong Dang, ${ }^{\dagger}$ Kapil Dev, $^{+}$Yuan Gao, $^{+}$Swee Tiam Tan, ${ }^{\dagger}$ Xiao Wei Sun, ${ }^{+, *}$ \\ and Hilmi Volkan Demir ${ }^{\dagger, \pm, s, *}$ \\ 'LUMINOUS! Centre of Excellence for Semiconductor Lighting and Displays, School of Electrical and Electronic Engineering, and ${ }^{\ddagger}$ School of Physical and Mathematical \\ Sciences, Nanyang Technological University, Nanyang Avenue, Singapore 639798, and ${ }^{\S}$ Department of Electrical and Electronics Engineering and Department \\ of Physics, UNAM-Institute of Materials Science and Nanotechnology, Bilkent University, Bilkent, Ankara, Turkey 06800. "Present address: Department of \\ Electrical-Electronics Engineering, Abdullah Gul University, Kayseri, Turkey 38039.
}

\begin{abstract}
Flexible information displays are key elements in future optoelectronic devices. Quantum dot light-emitting diodes (QLEDs) with advantages in color quality, stability, and cost-effectiveness are emerging as a candidate for singlematerial, full color light sources. Despite the recent advances in QLED technology, making high-performance flexible QLEDs still remains a big challenge due to limited choices of proper materials and device architectures as well as poor mechanical stability. Here, we show highly efficient, large-area QLED tapes emitting in red, green, and blue (RGB) colors with top-emitting design and polyimide tapes as

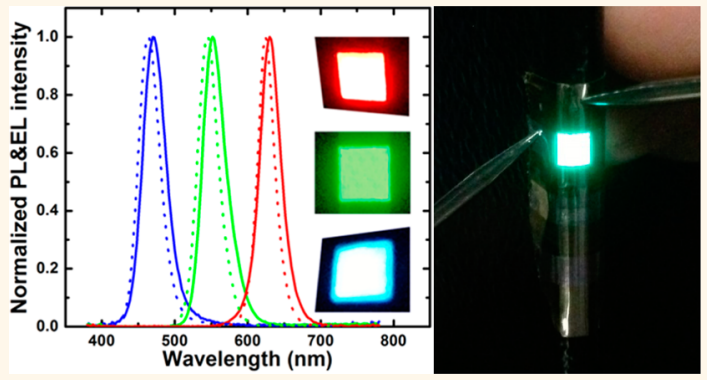
flexible substrates. The brightness and quantum efficiency are $20000 \mathrm{~cd} / \mathrm{m}^{2}$ and $4.03 \%$, respectively, the highest values reported for flexible QLEDs. Besides the excellent electroluminescence performance, these QLED films are highly flexible and mechanically robust to use as electrically driven light-emitting stickers by placing on or removing from any curved surface, facilitating versatile LED applications. Our QLED tapes present a step toward practical quantum dot based platforms for high-performance flexible displays and solid-state lighting.
\end{abstract}

KEYWORDS: quantum dot $\cdot$ polyimide tape $\cdot$ flexibility $\cdot$ electroluminescence $\cdot$ light-emitting diodes

$\mathrm{C}$ olloidal quantum dot light-emitting diodes (QLEDs) have recently received considerable attention for next-generation lighting and display systems due to their narrow emission line width, ${ }^{1-4}$ high brightness, ${ }^{5,6}$ color tunability, ${ }^{7,8}$ and cost-effective fabrication techniques. ${ }^{9-13}$ The strong quantum confinement effect in semiconductor nanocrystals allows one to engineer their emission color across the full visible spectrum just by changing their size in simple colloidal synthesis methods, realizing full color, single-material LEDs. Rapid progress has been made in QLEDs' performance during their two decades of development. ${ }^{14}$ Now, QLEDs are emerging as an undeniable competitor to organic light-emitting diodes (OLEDs) for lighting and display applications. ${ }^{15,16}$ QLEDs, typically using a QD film thickness of less than 100 nanometers, are very thin, making them virtually transparent and flexible, the key elements that OLEDs have successfully demonstrated for future information displays. ${ }^{17,18}$ However, challenges remain in the development of flexible QLEDs due to the specific requirements of electrodes and flexible substrates for device fabrication and operation. Indium tin oxide (ITO) is still the main electrode material for flexible and nonflexible QLEDs, ${ }^{19,20}$ but its brittle nature ${ }^{17}$ is not quite suitable for flexible optoelectronic devices. QLED fabrication involves multiple steps of solution-processed techniques with various chemicals and high-temperature annealing, therefore limiting the device architecture designs and the choices of suitable flexible plastic substrates. For example, aqueous polyethylene dioxythiophene:polystyrenesulfonate (PEDOT:PSS), which is widely used as a buffer layer for QLED fabrication, is generally annealed at $\sim 150{ }^{\circ} \mathrm{C}$, to remove solvents and functionalize this charge injection layer. ${ }^{21,22}$ Such high-temperature annealing usually causes the destruction or distortion of flexible substrates. In addition,

\section{* Address correspondence to volkan@stanfordalumni.org (H. V. Demir); exwsun@ntu.edu.sg (X.W. Sun).}

Received for review May 12, 2014 and accepted July 14, 2014.

Published online July 14, 2014 $10.1021 /$ nn502588k

(C) 2014 American Chemical Society 
(a)

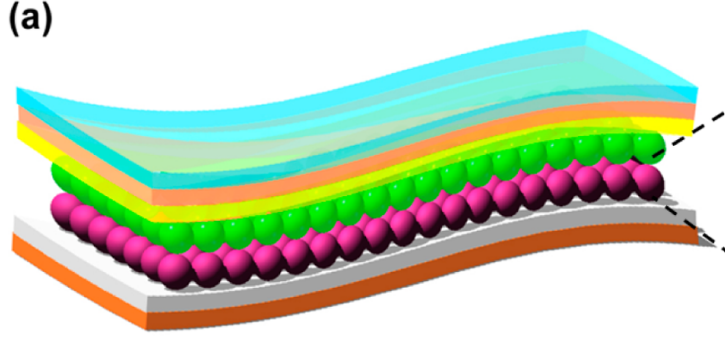

(b)
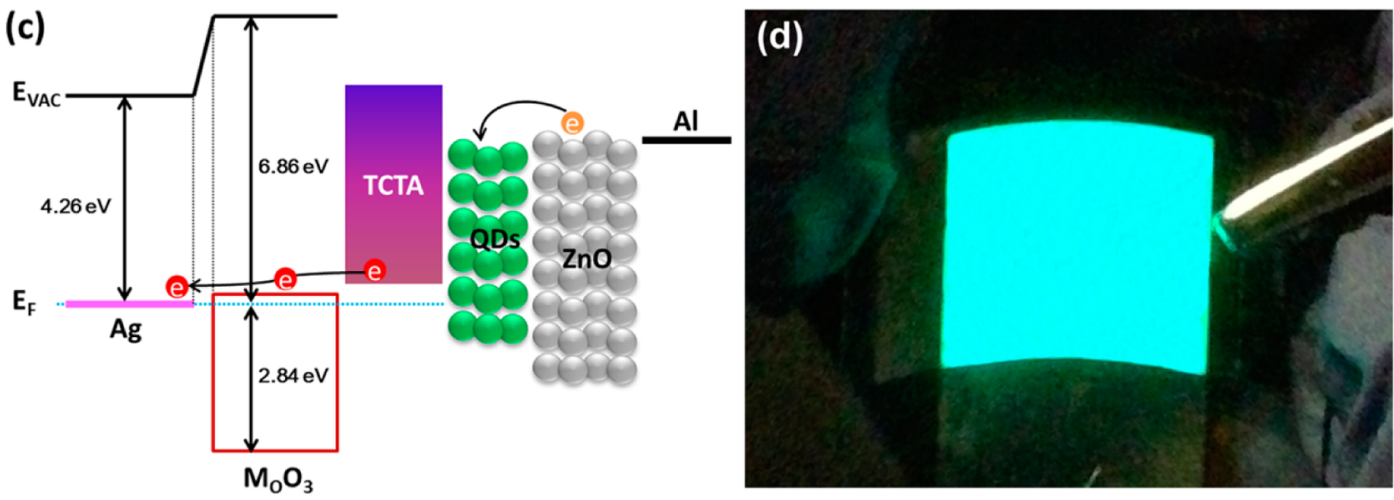

Figure 1. (a) Schematic of the QLED film configuration (from the bottom to the top): Kapton tape/AI/ZnO NPs/QDs/TCTA/ $\mathrm{MoO}_{3} / \mathrm{Ag}$. The CdSe/ZnS QD and ZnO NP layers were deposited by the spin-casting method, and other layers were deposited by thermal evaporation. (b) AFM image of spin-coated ZnO nanoparticle ETL formed on the Kapton tape/Al surface. (c) Energy level diagram of the QLED film. (d) Photographic image of a large-area QLED tape placed on a flexible plastic substrate $(20 \mathrm{~mm} \times 25 \mathrm{~mm})$.

only a few types of plastic such as poly(ethyleneterephthalate) (PET) can be used as flexible substrates because common solvents, e.g., toluene, often damage organic substrates. Relaxing these requirements to achieve flexible QLEDs results in greatly limited device performance.

Here, we report flexible top-emitting QLED tapes employing inverted device architecture with Kapton polyimide as the substrate and metallic thin films (Al, $\mathrm{Ag}$ ) as the electrodes. Highly flexible Kapton polyimide films are stable against many organic solvents and can withstand relatively high temperatures $\left(\sim 260{ }^{\circ} \mathrm{C}\right)$, which allowed us to optimize them for the best device performance. Such highly flexible top-emitting QLED films demonstrate maximum brightness and efficiency values of $20000 \mathrm{~cd} \mathrm{~m}^{-2}$ and $4.03 \%$, which are the highest performance values for flexible QLEDs reported to date. By controlling the quantum dot (QD) emission wavelength during colloidal synthesis, we achieved flexible QLED tapes with very high performance in all three colors: red, green, and blue (RGB). As an additional advantage of the Kapton tape (optionally one side with adhesive) and the mechanically robust structure, our QLED films can be easily placed on (and removed from) essentially any three-dimensional surface, even with a very large active area of $2 \mathrm{~cm} \times 2.5 \mathrm{~cm}$, the largest flexible QLED. The uses are akin to QLED stickers. These results demonstrate a practical route toward flexible QLEDs for information display applications.

\section{RESULTS AND DISCUSSION}

The top-emitting device architecture was demonstrated in inorganic and organic LEDs with advantages of enhanced aperture ratio of display. ${ }^{23}$ We first employed this architecture to design our flexible QLED films, as top-emitting LEDs are of increasing interest since the light outcoupling from the top allows LED devices on opaque substrates, which is quite useful for many applications such as high-resolution active matrix (AM) displays. ${ }^{24,25}$ The corresponding structure of our QLED films comprises a multilayer thin film architecture of Kapton tape/Al/ZnO nanoparticles (NPs)/ $\mathrm{QDs} / 2,2^{\prime}, 2^{\prime \prime}$-tris( $N$-carbazolyl)triphenylamine (TCTA)/ $\mathrm{MoO}_{3} / \mathrm{Ag}$, as shown in Figure 1a. The thickness of the Ag layer (anode) on top of the device was optimized at $18 \mathrm{~nm}$ to balance between conductivity and transmissibility for the best device performance. The CdSe/ZnS core-shell-structured QDs, prepared by a one-pot synthesis method, ${ }^{3}$ were used as the emissive layer. The $\mathrm{ZnO}$ nanoparticle film serving as the electron transport layer (ETL) was spin-casted from $\mathrm{ZnO}$ nanoparticles in butanol solution. Note that, although the high-quality $\mathrm{ZnO}$ nanoparticle films deposited on ITO have been achieved before, the present case is quite different with the $\mathrm{ZnO}$ nanoparticle film on Al. Because of the obvious distinction in their surface properties such as surface energy and hydrophobic/hydrophilic nature, ${ }^{26} \mathrm{ZnO}$ nanoparticle films form multiple small cracks and some large broken areas on Al (Supporting Information Figure S1). In our work, we achieved uniform $\mathrm{ZnO}$ nanoparticle films by the modification of the surface properties of ZnO NPs. The ZnO NPs employed here were prepared with an approach modified from that previously reported by a solution-precipitation process. ${ }^{9}$ The surface ligands of the ZnO NPs were removed 

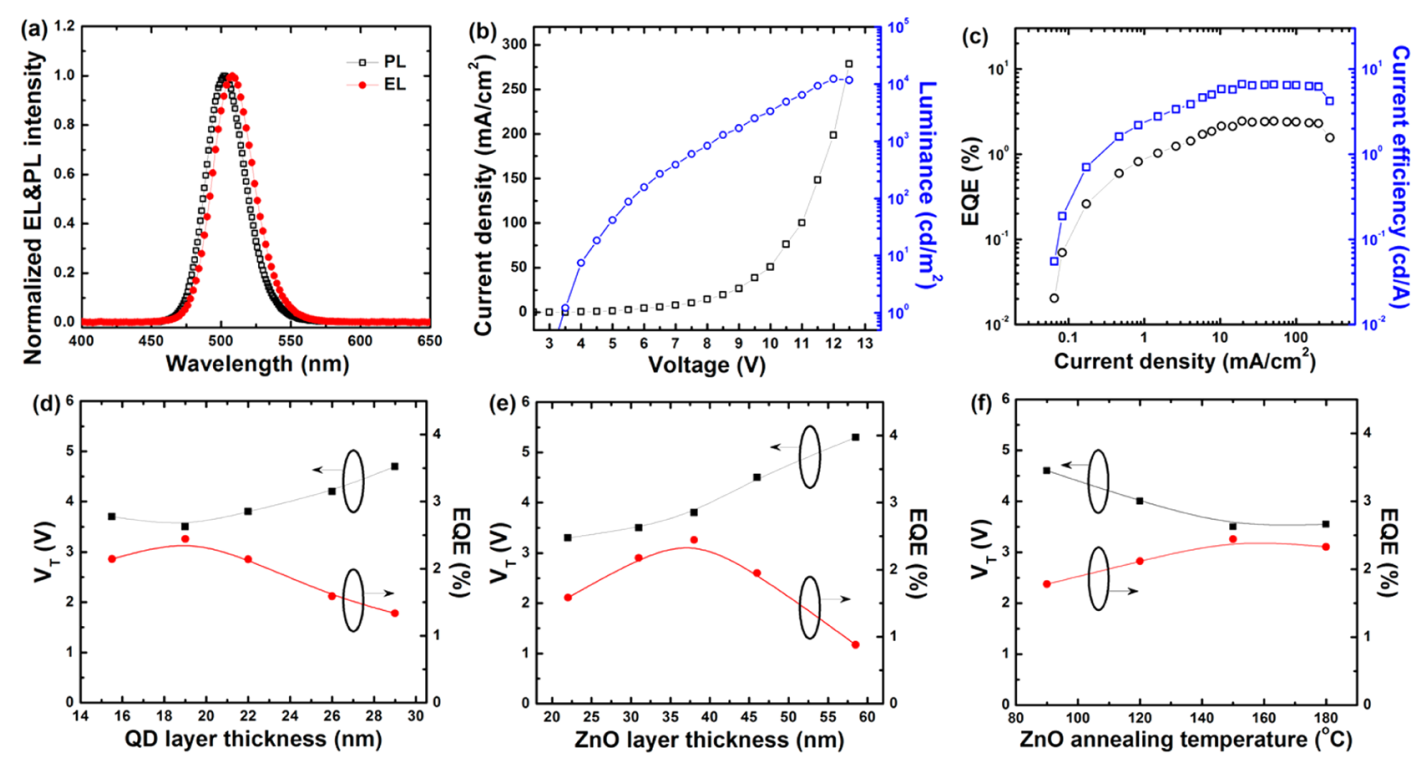

Figure 2. (a) Normalized PL and EL spectra of QDs and a typical QLED tape at an applied voltage of 6 V. (b) Current density and peak luminance of the QLED films as a function of bias voltage. (c) External quantum efficiency (EQE) and current efficiency (CE) of the QLED tapes as a function of current density. (d) Turn-on voltage $\left(V_{T}\right)$ and maximum EQE vs QD layer thickness ( $\mathrm{ZnO}$ nanoparticle layer thickness of $38 \mathrm{~nm}$ ). The turn-on voltage is defined as the voltage at which the luminance is $1 \mathrm{~cd} \mathrm{~m}^{-2}$. (e) $V_{\mathrm{T}}$ and maximum EQE vs ZnO nanoparticle layer thickness (QD layer thickness of $19 \mathrm{~nm}$ ). (f) $V_{\mathrm{T}}$ and maximum EQE vs $\mathrm{ZnO}$ nanoparticle layer annealing temperature (thickness of QD and $\mathrm{ZnO}$ nanoparticle layers is 19 and $38 \mathrm{~nm}$, respectively).

by adding excess precipitant solvent (hexane/ethanol), and then the resulting white precipitant was dispersed into butanol. As shown in Figure 1b, the surface roughness (RMS) of the film made from a $\mathrm{ZnO}$ nanoparticle butanol solution is only $3.6 \mathrm{~nm}$, about the size of $\mathrm{ZnO}$ nanoparticles and the RMS of Kapton tape (Supporting Information Figure S2), indicating excellent surface smoothness. The schematic energy level diagram of the device depicted in Figure 1c shows that the electrons can easily be injected from the Al to the QD layer via the conduction band of the $\mathrm{ZnO}$ nanoparticle layer. However, the case is different for the hole injection. ${ }^{27-29}$ For $n$-doped $\mathrm{MoO}_{3}$, owing to the deep lying electronic states of $\mathrm{MoO}_{3}$, the hole injection from anode to the organic semiconductor (TCTA) results from carrier breakthrough from the $\mathrm{MoO}_{3} / \mathrm{TCTA}$ junction. The electrons are extracted from the highest occupied molecular orbital (HOMO) level of TCTA through the conduction band of $\mathrm{MoO}_{3}$. Figure 1d displays bright, uniform, and saturated green QD emission from our flexible QLED film with a surface area of $2 \mathrm{~cm} \times 2.5 \mathrm{~cm}$, which is the largest area reported for flexible QLEDs so far. The uniform size of QDs and ZnO nanoparticles and no bubbles in the Kapton tape substrate in the device fabrication process are required for obtaining such large-area flexible devices.

Figure $2 \mathrm{a}$ presents the electroluminescence (EL) spectrum of our typical green QLED tapes. The pure QD emission at a peak wavelength of $\sim 508 \mathrm{~nm}$ with a full width at half-maximum (fwhm) of $34 \mathrm{~nm}$ illustrates the highly efficient recombination of electrons and holes in the QD emissive layer. The peak wavelength is red-shifted $(\sim 6 \mathrm{~nm})$ from the QD solution's photoluminescence $(\mathrm{PL})$ resulting from a combination of finite dot-to-dot interactions in close-packed solid films and the electric-field-induced Stark effect. The typical current density and luminance curves as a function of applied voltage for the unpackaged QLED films are shown in Figure $2 \mathrm{~b}$. The maximum luminance reaches $12300 \mathrm{~cd} / \mathrm{m}^{2}$, and the turn-on voltage $\left(V_{T}\right)$ of $3.5 \mathrm{~V}$ is relatively low, suggesting a small barrier height for the charge injection into the QDs. In addition, the external quantum efficiency-current efficiency-current density (EQE-CE-J) characteristics of the QLED film exhibit high device efficiency, with a maximum EQE of $2.44 \%$ and CE of $6.61 \mathrm{~cd} / \mathrm{A}$ (Figure 2c). The ZnO nanoparticles as ETLs in the inverted QLEDs help to neutralize QDs, i.e., turning dark (charged) QDs to bright (neutral) QDs, and, therefore, improve the device performance. ${ }^{1}$ But like other metal oxides, $\mathrm{ZnO}$ also quenches the quantum yield of QDs due to an ultrafast nonradiative process that depends on the proximity of emitters to the interface. The two opposite effects imply that the device performance is strongly dependent on the thickness of the QD and ZnO nanoparticle layers. ${ }^{9}$ Figure $2 \mathrm{~d}$ and e show the turn-on voltage $V_{\mathrm{T}}$ and maximum EQE as a function of the QD and $\mathrm{ZnO}$ nanoparticle layer thickness for the QLED tapes. With the $\mathrm{ZnO}$ layer fixed at $\sim 38 \mathrm{~nm}$, the lowest $V_{\mathrm{T}}$ of $3.5 \mathrm{~V}$ and the maximum EQE of $2.44 \%$ can be achieved when the device has a $19 \mathrm{~nm}$ thick QD layer (Figure 2d), which is equivalent to three QD monolayers. This result is consistent with the previous reports that a few monolayers of QDs are needed for efficient recombination of electrons and holes to provide exciton formation in the QDs. ${ }^{15}$ For a $19 \mathrm{~nm}$ thick QD layer, 
increasing the $\mathrm{ZnO}$ layer thickness from $20 \mathrm{~nm}$ to $60 \mathrm{~nm}$ resulted in a continuous increase of $V_{\mathrm{T}}$ from $3.3 \mathrm{~V}$ to $5.3 \mathrm{~V}$, while the highest EQE value is achieved when the $\mathrm{ZnO}$ thickness is $38 \mathrm{~nm}$ (Figure 2e). This implies that a finite voltage is needed across the $\mathrm{ZnO}$ layer, making the efficiency of the device with a thicker $\mathrm{ZnO}$ nanoparticle layer dramatically decrease. In addition to the above factors, we found that the annealing treatment of the $\mathrm{ZnO}$ nanoparticle film to reduce the defects can also improve the device performance significantly. As shown in Figure 2f, for a QLED tape with a $19 \mathrm{~nm}$ thick QD layer and a $38 \mathrm{~nm}$ thick $\mathrm{ZnO}$ nanoparticle layer, increasing the $\mathrm{ZnO}$ annealing temperature from $90^{\circ} \mathrm{C}$ to $180^{\circ} \mathrm{C}$, decreases the $V_{T}$ steadily until $150^{\circ} \mathrm{C}$ and the EQE increases to the maximum level of $2.44 \%$ at $150^{\circ} \mathrm{C}$. After that, the EQE and $V_{\mathrm{T}}$ are almost unchanged with increasing annealing temperature. We believe that this is enabled by enhancing the electron transport in $\mathrm{ZnO}$ nanoparticle films, which is associated with the reduction in defect concentration of $\mathrm{ZnO}$ nanoparticles.

To further confirm the effect of defects in $\mathrm{ZnO}$ nanoparticles in the device's EL performance, we compared the PL intensity of $\mathrm{ZnO}$ nanoparticle films annealed at different temperatures with an excitation source of $300 \mathrm{~nm}$ wavelength. As shown in Figure 3, the PL spectra of $\mathrm{ZnO}$ nanoparticles consist of a sharp UV emission

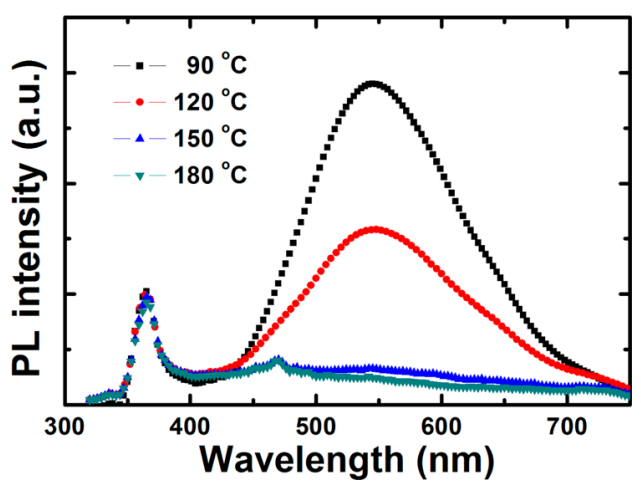

Figure 3. Room-temperature PL spectra for $\mathrm{ZnO}$ nanoparticle films annealed at different temperatures from 90 to $180^{\circ} \mathrm{C}$. band and a broad green emission band peaking at $550 \mathrm{~nm}$. The narrow UV emission is attributed to the radiative annihilation of excitons, ${ }^{30}$ while the broad visible emission is associated with defects in $\mathrm{ZnO}$ nanoparticles. ${ }^{31}$ The origin of defect emission has been the subject of ongoing debate, and the widely accepted theory is that they are caused by the oxygen vacancies or zinc interstitials. ${ }^{32,33}$ In addition, it can be noted that the PL intensities of the broad green emission band dramatically decrease with the annealing temperature increasing from $90^{\circ} \mathrm{C}$ to $150^{\circ} \mathrm{C}$. Beyond $150^{\circ} \mathrm{C}$, there is no obvious green emission observed from $\mathrm{ZnO}$. In contrast, the UV emission intensities vary only slightly, suggesting that the total defect concentration in $\mathrm{ZnO}$ is significantly decreased with increasing annealing temperature. Combined with the observed EQE and $V_{T}$ trends in the case of QLED films, these results show a good correlation between the device EL performance and defect concentration in $\mathrm{ZnO}$ nanoparticles. The significant reduction in the defect concentration and increase in the crystal quality of $\mathrm{ZnO}$ nanoparticles improve the electron transport due to the decrease in defect-trapped electrons in ZnO (Supporting Information Figure S3), resulting in enhanced EL intensity. It should be noted that the annealing temperature of $150{ }^{\circ} \mathrm{C}$ is too high for most flexible substrates, which may limit the QLED device performance to a great extent, while this is fine for Kapton polyimide films.

The thin Ag layer ( $18 \mathrm{~nm}$ ) serves as a semitransparent anode in our top-emitting QLED tapes, and an organic capping (OC) layer on top of the Ag layer could modify the device's optical structure as the change of interference effects within the device. ${ }^{34}$ The OC layer has been demonstrated to improve the outcoupling efficiency of the top-emitting OLED. ${ }^{35}$ Here, we deposited a tris-8-hydroxyquinoline aluminum $\left(\mathrm{Alq}_{3}\right)$ film as a capping layer above the top contact. Figure 4 shows the performance of the device with the $\mathrm{OC}$ layer as measured in the vertical direction. We improved the device performance significantly just by adding the optical outcoupling $\mathrm{Alq}_{3}$
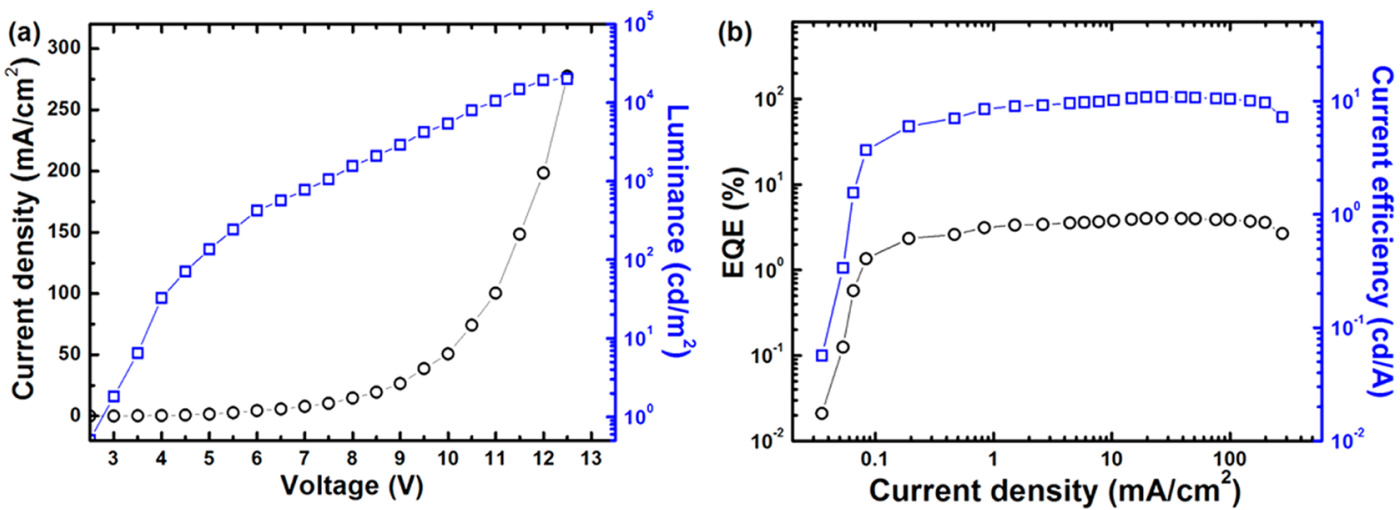

Figure 4. (a) Current density and luminance of QLED tape as a function of applied voltage. (b) External quantum efficiency and current efficiency of QLED tape as a function of current density. 

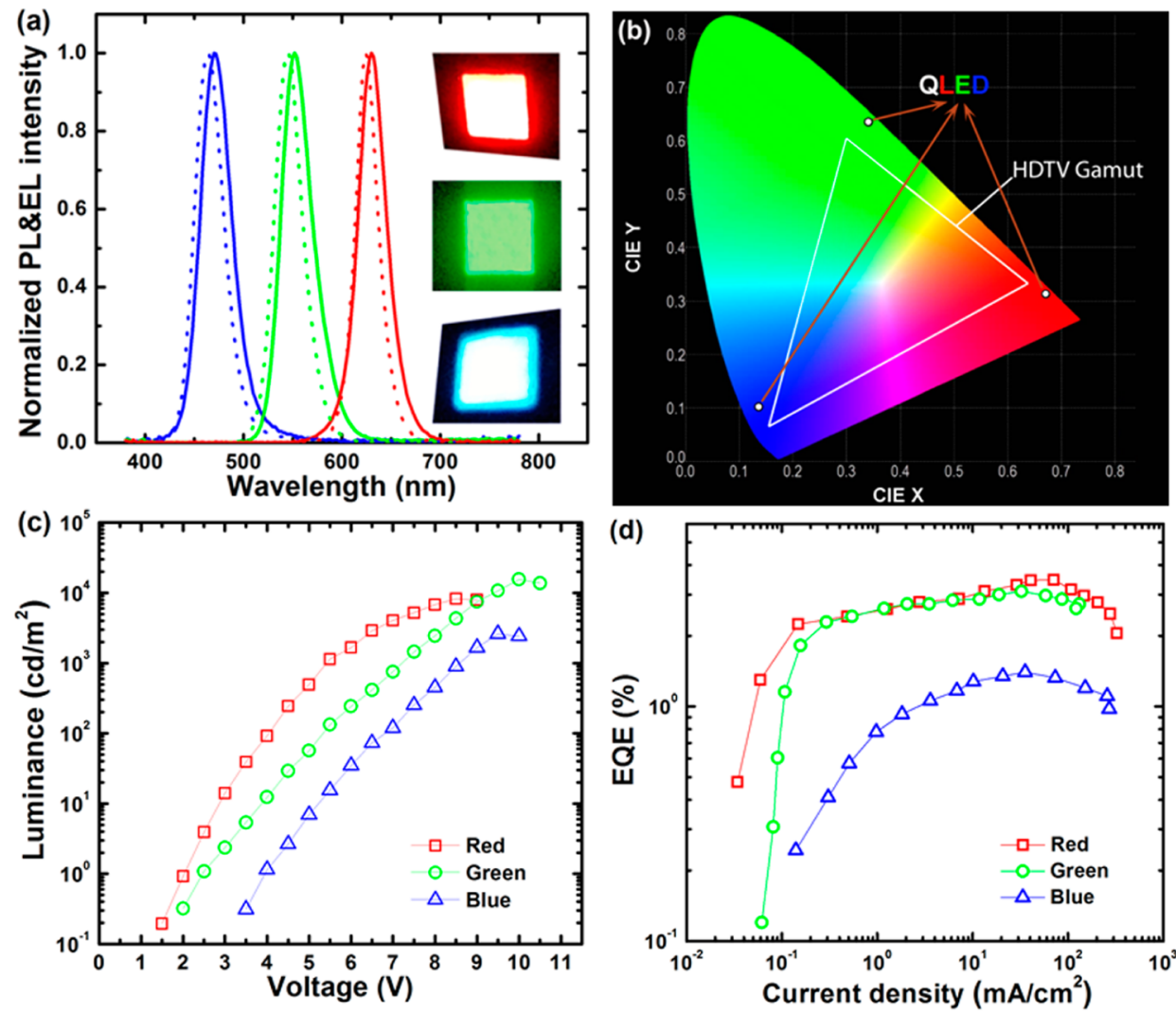

Figure 5. (a) Normalized EL spectra of QLED films with peak emission wavelengths of $470 \mathrm{~nm}$ (blue), $551 \mathrm{~nm}$ (yellowish green), and $630 \mathrm{~nm}$ (red). Inset: Photographic images of the RGB QLED tapes. (b) CIE coordinates of the three devices together with the HDTV color standards. (c) Luminance of our RGB QLED films as a function of applied voltage. (d) External quantum efficiency of QLED tapes as a function of current density.

layer with a film thickness of $40 \mathrm{~nm}$. The maximum brightness reaches $20000 \mathrm{~cd} / \mathrm{m}^{2}$ (Figure 4a), and an EQE level of $4.03 \%$ and a current efficiency value of $10.9 \mathrm{~cd} / \mathrm{A}$ can be achieved at a current density of $26.6 \mathrm{~mA} / \mathrm{cm}^{2}$ (Figure $4 \mathrm{~b}$ ). The thin OC layer improves the efficiency and the brightness of our flexible QLED film by $165 \%$ and $162 \%$, respectively.

Using our optimized structure with multicolored quantum dots, we obtained RGB-emitting QLED tapes. Figure 5a presents the normalized EL spectra of these QLED films with three different peak emission wavelengths $(470,551$, and $630 \mathrm{~nm})$ at the applied voltage of $6 \mathrm{~V}$. It can be observed that all the EL spectra are saturated with QD emission and are slightly red-shifted $(5-6 \mathrm{~nm})$ from the corresponding PL spectra of QD solutions. The fwhm is 33,37 , and $37 \mathrm{~nm}$ for the respective RGB colors. The inset shows photographic images of the three operating devices, which display nearly saturated color emission. As shown by the Commission Internationale de Eclairage (CIE) chromaticity diagram in Figure 5b, these QLED films cover a larger area than a high-definition television (HDTV) standard color triangle. ${ }^{15}$ The luminance and EQE as a function of the current density for the optimized QLED films are shown in Figure $5 \mathrm{c}$ and $\mathrm{d}$. The maximum luminance and $E Q E$ values of $2600 \mathrm{~cd} \mathrm{~m}^{-2}$ and $1.40 \%$ for the blue emission (peak at $470 \mathrm{~nm}$ ), $15600 \mathrm{~cd} \mathrm{~m}^{-2}$
TABLE 1. Summary of the Optical and Electrical Properties of QDs and QLED Tapes

\begin{tabular}{|c|c|c|c|c|c|c|c|}
\hline color of QLED & $\begin{array}{c}\mathrm{PL} \lambda_{\max } \\
(\mathrm{nm})\end{array}$ & $\begin{array}{c}\text { EL } \lambda_{\text {max }} \\
(\mathrm{nm})\end{array}$ & $\begin{array}{l}\text { fwhm } \\
(n m)\end{array}$ & $\begin{array}{l}V_{\mathrm{T}} \\
(\mathrm{V})\end{array}$ & $\begin{array}{c}\mathrm{EQE}_{\text {max }} \\
(\%)\end{array}$ & $L_{\max }\left(\mathrm{cd} / \mathrm{m}^{2}\right)$ & $\begin{array}{c}\text { CIE index } \\
(x, y)\end{array}$ \\
\hline blue & 465 & 470 & 37 & 3.9 & 1.40 & 2600 & $0.135,0.103$ \\
\hline green & 502 & 508 & 34 & 3 & 4.03 & 20000 & $0.086,0.643$ \\
\hline yellowish-green & 545 & 551 & 37 & 2.5 & 3.09 & 15600 & $0.335,0.631$ \\
\hline red & 624 & 630 & 33 & 2 & 3.46 & 8200 & $0.682,0.315$ \\
\hline
\end{tabular}

and $3.09 \%$ for the yellowish green emission (at $551 \mathrm{~nm}$ ), and $8200 \mathrm{~cd} \mathrm{~m}^{-2}$ and $3.46 \%$ for the red emission (at $630 \mathrm{~nm}$ ) were achieved. Table 1 summarizes the detailed performance parameters of our RGB QLED tapes. The maximum luminance and efficiency are the highest values reported to date for correspondingly colored flexible QLEDs. Nevertheless, it still can be expected that further optimization such as improving the quantum yields of QD films would realize better device performances.

In addition to the optoelectrical high performance, the distinct advantages of our top-emitting QLED tapes over previous flexible QLEDs are their highly flexible and mechanically robust structure. With the use of Kapton tape, our QLED stickers are easily placed on or removed from almost any three-dimensional surface, which facilitates the versatile use of our QLED platform. 

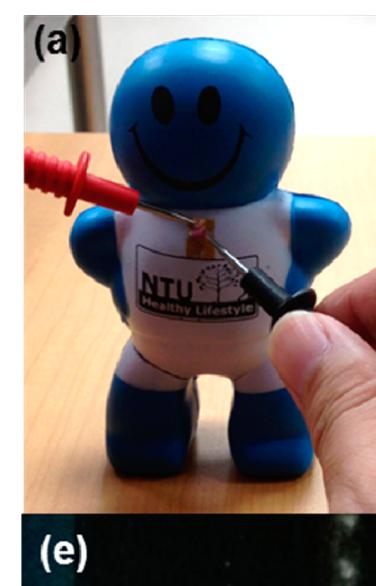
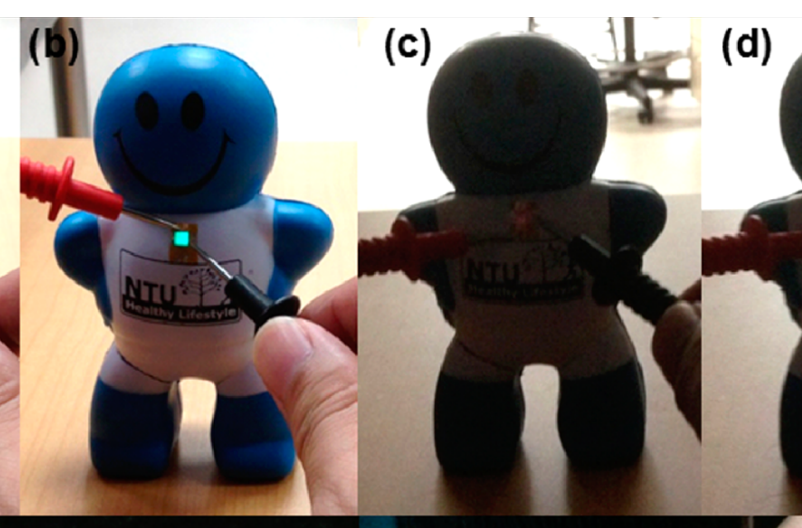

(d)

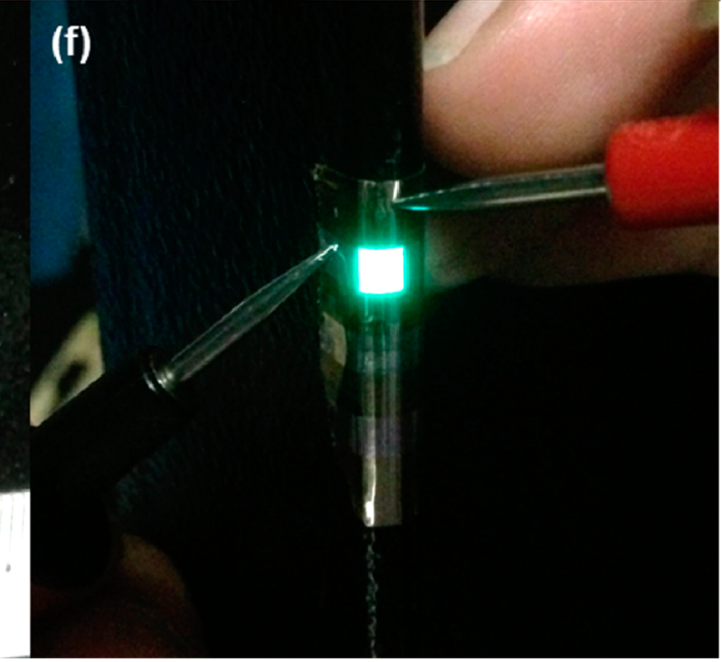

Figure 6. Photographic images of a QLED tape with a pixel size of $3 \mathrm{~mm} \times 3 \mathrm{~mm}$ placed on different nonplanar surfaces. (a, b) "Off" and "On" device placed on a mascot in a bright room, respectively. (c, d) "Off" and "On" device placed on the mascot in a dark room, respectively. $(\mathrm{e}, \mathrm{f})$ "Off" and "On" device placed on a thin steel plate under room light, respectively.

As shown in Figure $6 a-d$, the green-emitting QLED tape, stuck on a mascot, works very well with high brightness under room light or dark conditions. Furthermore, the operating QLED film can be bent arbitrarily and is free of cracks and dark spots even under almost completely folded bending. As shown in Figure 6e,f, the working QLED tape is placed and bent around a very thin steel plate. Its brightness and uniformity do not show any degradation. The excellent mechanical robustness of our QLED tapes allows us to use them as light-emitting stickers, i.e., a luminescent sticker for three-dimensional surfaces. Its robustness is further confirmed by the device bending test (Supporting Information Figure S4), The brightness remains at $90 \%$ after being bent 300 times on a $4 \mathrm{~mm}$ diameter rod, whereas with bending on a $12 \mathrm{~mm}$ diameter rod there is almost no obvious change in the brightness even after repeating 500 times.

\section{CONCLUSION}

In conclusion, we have demonstrated highly flexible, large-area, full-color, top-emitting QLED tapes with record high efficiency in flexible QLEDs. These superior performances were achieved by the efficient design of a top-emitting device architecture with the proper choice of Kapton tape as the flexible substrate, the surface modification of $\mathrm{ZnO}$ nanoparticles for an efficient electron-transporting layer, and the deposition of an $\mathrm{Alq}_{3}$ capping layer as an outcoupling element. These large-area, ITO-free QLED films also show excellent mechanical robustness and can be used as electrically driven light-emitting stickers on any three-dimensional surface, which enables the extensive and versatile use of LEDs. Our results illustrate a further step toward the practical application of flexible QLEDs in optoelectronic devices including flexible, fullcolor information displays and surface lighting.

\section{METHODS}

Materials. Cadmium oxide (CdO; 99.99+\%, Aldrich), zinc acetate $\left(\mathrm{Zn}(\text { Acet })_{2} ; 99.99 \%\right.$, Aldrich), oleic acid (OA; 90\%, Aldrich), 1-octadecene (1-ODE; 90\%, Aldrich), tri- $n$-octylphosphine
(TOP, 90\%, Aldrich), selenium (Se; 99.99\%, Aldrich), sulfur (S; 99.5+\%, Sigma-Aldrich), fluorescein-27 (90+\%, Sigma), dimethyl sulfoxide (DMSO; 99.98\%, Fisher Scientific UK Limited), tetramethylammonium hydroxide pentahydrate ( $97 \%$, Sigma), 
methanol (AR), ethanol (AR), butanol (AR), hexane (AR), acetone $(A R)$, and toluene $(A R)$ were used as received without further purification. The TCTA, $\mathrm{Alq}_{3}$, and $\mathrm{MoO}_{3}$ used for device fabrication were purchased from Luminescence Technology Corp. Kapton tape substrate was purchased from Dakin Engineering Pte Ltd.

Synthesis of Multicolored QDs and Zn0 Nanoparticles. Green- and blue-emitting CdSe/ZnS QDs with chemical composition gradients were prepared using a one-pot synthesis method, ${ }^{3}$ and the red-emitting $\mathrm{CdSe} / \mathrm{CdS} / \mathrm{ZnS}$ core-shell-structured QDs were prepared by the multiple injection method according to the same literature. For a typical preparation of green-emitting QDs, $0.1 \mathrm{mmol}$ of cadmium oxide, $4 \mathrm{mmol}$ of zinc acetate, and $5 \mathrm{~mL}$ of oleic acid were loaded in a $50 \mathrm{~mL}$ three-neck flask and heated to $150{ }^{\circ} \mathrm{C}$ under vacuum to form cadmium oleate $\left(\mathrm{Cd}(\mathrm{OA})_{2}\right)$ and zinc oleate $\left(\mathrm{Zn}(\mathrm{OA})_{2}\right)$. Then $20 \mathrm{~mL}$ of 1 -octadecene was added to the reaction flask, and the reactor was then filled with nitrogen and heated to $300{ }^{\circ} \mathrm{C}$. At the elevated temperature, $1.6 \mathrm{~mL}$ of tri- $n$-octylphosphine with $0.15 \mathrm{mmol}$ of selenium and $4 \mathrm{mmol}$ of sulfur was injected into the flask swiftly, and the reaction mixture was maintained at $300{ }^{\circ} \mathrm{C}$ for 10 min for the QD growth. To purify the synthesized QDs, the reaction mixture was cooled to room temperature, and the QDs were extracted by the addition of acetone and methanol, followed by centrifugation. The as-prepared QDs were readily dispersed in toluene. The purified QDs with an average particle size of $\sim 7 \mathrm{~nm}$ were measured to give a quantum yield of $\sim 60 \%$ in toluene by comparing the fluorescence intensities with a standard reference dye, fluorescein- 27.

$\mathrm{ZnO}$ nanoparticles with a typical mean diameter of 3-4 nm were synthesized based on a solution-precipitation process. ${ }^{9}$ For a typical synthesis, zinc acetate in DMSO $(0.1 \mathrm{M}, 20 \mathrm{~mL})$ and tetramethylammonium hydroxide in ethanol $(0.55 \mathrm{M}, 20 \mathrm{~mL})$ were mixed and stirred for $1 \mathrm{~h}$ in ambient air, then washed at least four times with excess precipitant solvents such as hexane/ ethanol (1:4 by volume), and then the as-prepared white precipitant was dispersed in butanol at a concentration of $\sim 25 \mathrm{mg} \mathrm{mL}^{-1}$.

Fabrication of QLED Devices. The Kapton tape was wiped by cotton tips with DI-water and acetone first, and the Al cathode $(190 \mathrm{~nm})$ was then deposited on top. The $\mathrm{ZnO}$ nanoparticle layer was then spin-coated on the Al cathode deposited Kapton tape from a $\mathrm{ZnO}$ butanol solution at $500-6000 \mathrm{rpm}$ for $60 \mathrm{~s}$ and annealed at $150^{\circ} \mathrm{C}$ for $30 \mathrm{~min}$ in a nitrogen glovebox. Next, the QD layer was deposited on the ZnO NPs layer by spin-coating the QD dispersion (QDs were dispersed in toluene at $15 \mathrm{mg} / \mathrm{mL}$ ) at a rate of $1000-6000 \mathrm{rpm}$ for $60 \mathrm{~s}$ and cured at $90{ }^{\circ} \mathrm{C}$ for $30 \mathrm{~min}$. After that, the TCTA $(60 \mathrm{~nm}), \mathrm{MoO}_{3}(10 \mathrm{~nm})$, and $\mathrm{Ag}$ $(18 \mathrm{~nm})$ layers were thermally deposited under a base pressure of $\sim 2 \times 10^{-4} \mathrm{~Pa}$.

Instrumentation. The EL spectra of the flexible QLED films were measured using a Photo Research PR705 Spectra Scan spectrometer, while the luminance-current-voltage characteristics of the devices were measured simultaneously with a programmable Yokogawa GS610 source meter and a Konica Minolta LS-110 luminance meter in air at room temperature. Atomic force microscopy (Cypher AFM, Asylum Research) was used to characterize the mophology of the $\mathrm{ZnO}$ nanoparticle films. The PL spectra were recorded using a Shimadzu RF-5301PC spectrofluorophotometer equipped with a $150 \mathrm{~W}$ xenon lamp as the excitation source. A four-point probe instrument (CMTSR2000N) was employed to measure the conductivity of the $\mathrm{ZnO}$ nanoparticle films. All measurements were carried out at room temperature under ambient conditions.

Conflict of Interest: The authors declare no competing financial interest.

Acknowledgment. The authors would like to acknowledge the financial support from Singapore National Research Foundation under NRF-RF-2009-09, NRF-CRP-6-2010-02, NRF-CRP11-2012-01, and NRF-CRP-11-2012-01 and the Science and Engineering Research Council, Agency for Science, Technology and Research (A*STAR) of Singapore (project nos. 0921010057 and 112120 2009).
Supporting Information Available: Digital optical microscopic images of $\mathrm{ZnO}$ nanoparticle films deposited on Al electrodes, AFM image of Kapton tape surface, electrical conductivities of ZnO nanoparticle films, and bending test of QLED films. This material is available free of charge via the Internet at http://pubs.acs.org

\section{REFERENCES AND NOTES}

1. Mashford, B. S.; Stevenson, M.; Popovic, Z.; Hamilton, C.; Zhou, Z.; Breen, C.; Steckel, J.; Bulovic, V.; Bawendi, M.; CoeSullivan, S.; et al. High-Efficiency Quantum-Dot LightEmitting Devices with Enhanced Charge Injection. Nat. Photonics 2013, 7, 407-412.

2. Cho, K. S.; Lee, E. K.; Joo, W. J.; Jang, E.; Kim, T. H.; Lee, S. J.; Kwon, S. J.; Han, J. Y.; Kim, B. K.; Choi1, B. L.; et al. HighPerformance Crosslinked Colloidal Quantum-Dot LightEmitting Diodes. Nat. Photonics 2009, 3, 341-345.

3. Bae, W. K.; Kwak, J.; Lim, J.; Lee, D.; Nam, M. K.; Char, K.; Lee, C.; Lee, S. Multicolored Light-Emitting Diodes Based on All-Quantum-Dot Multilayer Films Using Layer-by-Layer Assembly Method. Nano Lett. 2010, 10, 2368-2373.

4. Lee, K.-H.; Lee, J.-H.; Song, W.-S.; Ko, H.; Lee, C.; Lee, J.-H.; Yang, H. Highly Efficient, Color-Pure, Color-Stable Blue Quantum Dot Light-Emitting Devices. ACS Nano 2013, 7, 7295-7302.

5. Kwak, J.; Bae, W. K.; Lee, D.; Park, I.; Lim, J.; Park, M.; Cho, H.; Woo, H.; Yoon, D. Y.; Char, K.; et al. Bright and Efficient FullColor Colloidal Quantum Dot Light-Emitting Diodes Using an Inverted Device Structure. Nano Lett. 2012, 12, 23622366.

6. Lee, K.-H.; Lee, J.-H.; Kang, H.-D.; Park, B.; Kwon, Y.; Ko, H.; Lee, C.; Lee, J.; Yang, H. Over 40 cd/A Efficient Green Quantum Dot Electroluminescent Device Comprising Uniquely Large-Sized Quantum Dots. ACS Nano 2014, 8, 4893-4901.

7. Yang, X.; Zhao, D.; Leck, K. S.; Tan, S. T.; Tang, Y. X.; Zhao, J.; Demir, H. V.; Sun, X. W. Full Visible Range Covering InP/ZnS Nanocrystals with High Photometric Performance and Their Application to White Quantum Dot Light-Emitting Diodes. Adv. Mater. 2012, 24, 4180-4185.

8. Anikeeva, P. O.; Halpert, J. E.; Bawendi, M. G.; Bulović, V. Quantum Dot Light-Emitting Devices with Electroluminescence Tunable over the Entire Visible Spectrum. Nano Lett. 2009, 9, 2532-2536.

9. Qian, L.; Zheng, Y.; Xue, J.; Holloway, P. H. Stable and Efficient Quantum-Dot Light-Emitting Diodes Based on Solution-Processed Multilayer Structures. Nat. Photonics 2011, 5, 543-548.

10. Zhang, Y.; Xie, C.; Su, H.; Liu, J.; Pickering, S.; Wang, Y.; Yu, W. W.; Wang, J.; Wang, Y.; Hahm, J.; et al. Employing Heavy Metal-Free Colloidal Quantum Dots in Solution-Processed White Light-Emitting Diodes. Nano Lett. 2011, 11, 329-332.

11. Schreuder, M. A.; Xiao, K.; Ivanov, I. N.; Weiss, S. M.; Rosenthal, S. J. White Light-Emitting Diodes Based on Ultrasmall CdSe Nanocrystal Electroluminescence. Nano Lett. 2010, 10, 573-576.

12. Tan, Z.; Zhang, Y.; Xie, C.; Su, H.; Liu, J.; Zhang, C.; Dellas, N.; Mohney, S. E.; Wang, Y.; Wang, J.; et al. Near-Band-Edge Electroluminescence from Heavy-Metal-Free Colloidal Quantum Dots. Adv. Mater. 2011, 23, 3553-3558.

13. Zhang, X.; Zhang, Y.; Wang, Y.; Kalytchuk, S.; Kershaw, S. V.; Wang, Y.; Wang, P.; Zhang, T.; Zhao, Y.; Zhang, H.; et al. Color-Switchable Electroluminescence of Carbon Dot Light-Emitting Diodes. ACS Nano 2013, 7, 11234-11241.

14. Colvin, V. L.; Schlamp, M. C.; Alivisatos, A. P. Light-Emitting Diodes Made from Cadmium Selenide Nanocrystals and a Semiconducting Polymer. Nature 1994, 370, 354-357.

15. Shirasaki, Y.; Supran, G. J.; Bawendi, M. G.; Bulović, V. Emergence of Colloidal Quantum-Dot Light-Emitting Technologies. Nat. Photonics 2013, 7, 13-23.

16. Talapin, D. V.; Lee, J. S.; Kovalenko, M. V.; Shevchenko, E. V. Prospects of Colloidal Nanocrystals for Electronic and Optoelectronic Applications. Chem. Rev. 2010, 110, 389458. 
17. Han, T.-H.; Lee, Y.; Choi, M.-R.; Woo, S.-H.; Bae, S.-H.; Hong, B. H.; Ahn, J.-H.; Lee, T.-W. Extremely Efficient Flexible Organic Light-Emitting Diodes with Modified Graphene Anode. Nat. Photonics 2012, 6, 105-110.

18. Wang, Z. B.; Helander, M. G.; Qiu, J.; Puzzo, D. P.; Greiner, M. T.; Hudson, Z. M.; Wang, S.; Liu, Z. W.; Lu, Z. H. Unlocking the Full Potential of Organic Light-Emitting Diodes on Flexible Plastic. Nat. Photonics 2011, 5, 753-757.

19. Kim, T. H.; Cho, K. S.; Lee, E. K.; Lee, S. J.; Chae, J.; Kim, J. W.; Kim, D. H.; Kwon, J.-Y.; Amaratunga, G.; Lee, S. Y.; et al. Full-Colour Quantum Dot Displays Fabricated by Transfer Printing. Nat. Photonics 2011, 5, 176-182.

20. Tan, Z.; Xu, J.; Zhang, C.; Zhu, T.; Zhang, F.; Hedrick, B.; Pickering, S.; Wu, J.; Su, H.; Gao, S.; et al. Colloidal Nanocrystal-Based Light-Emitting Diodes Fabricated on Plastic Toward Flexible Quantum Dot Optoelectronics. J. Appl. Phys. 2009, 105, 034312.

21. Leck, K. S.; Divayana, Y.; Zhao, D.; Yang, X.; Abiyasa, A. P.; Mutlugun, E.; Gao, Y.; Liu, S.; Tan, S. T.; Sun, X. W.; et al. Quantum Dot Light-Emitting Diode with Quantum Dots Inside the Hole Transporting Layers. ACS Appl. Mater. Interfaces 2013, 5, 6535-6540.

22. Yang, X.; Mutlugun, E.; Zhao, Y.; Gao, Y.; Leck, K. S.; Ma, Y.; Ke, L.; Tan, S. T.; Demir, H. V.; Sun, X. W. Solution Processed Tungsten Oxide Interfacial Layer for Efficient HoleInjection in Quantum Dot Light-Emitting Diodes. Small 2014, 10, 247-252.

23. Hofmann, S.; Thomschke, M.; Lüssem, B.; Leo, K. TopEmitting Organic Light-Emitting Diodes. Opt. Express 2011, 19, 1250-1264.

24. Thomschke, M.; Reineke, S.; Lüssem, B.; Leo, K. Highly Efficient White Top-Emitting Organic Light-Emitting Diodes Comprising Laminated Microlens Films. Nano Lett. 2012, 12, 424-428.

25. Huang, Q.; Walzer, K.; Pfeiffer, M.; Lyssenko, V.; He, G.; Leo, K. Highly Efficient Top Emitting Organic Light-Emitting Diodes with Organic Outcoupling Enhancement Layers. Appl. Phys. Lett. 2006, 88, 113515.

26. Ma, H.; Yip, H.-L.; Huang, F.; Jen, A. K.-Y. Interface Engineering for Organic Electronics. Adv. Funct. Mater. 2010, 20, 1371-1388.

27. Meyer, J.; Khalandovsky, R.; Görrn, P.; Kahn, $\mathrm{A} . \mathrm{MoO}_{3}$ Films Spin-Coated from a Nanoparticle Suspension for Efficient Hole-Injection in Organic Electronics. Adv. Mater. 2011, 23, 70-73.

28. Murase, S.; Yang, Y. Solution Processed $\mathrm{MoO}_{3}$ Interfacial Layer for Organic Photovoltaics Prepared by a Facile Synthesis Method. Adv. Mater. 2012, 24, 2459-2462.

29. Kröger, M.; Hamwi, S.; Meyer, J.; Riedl, T.; Kowalsky, W.; Kahn, A. Role of the Deep-Lying Electronic States of $\mathrm{MoO}_{3}$ in the Enhancement of Hole-Injection in Organic Thin Films. Appl. Phys. Lett. 2009, 95, 123301.

30. Haranath, D.; Sahai, S.; Joshi, P. Tuning of Emission Colors in Zinc Oxide Quantum Dots. Appl. Phys. Lett. 2008, 92, 233113.

31. Lin, B.; Fu, Z.; Jia, Y.; Liao, G. Defect Photoluminescence of Undoping ZnO Films and Its Dependence on Annealing Conditions. J. Electrochem. Soc. 2001, 148, 110-113.

32. McCluskey, M. D.; Jokela, S. J. Defects in ZnO. J. Appl. Phys. 2009, 106, 071101.

33. Djurišić, A. B.; Choy, W. C. H.; Roy, V. A. L.; Leung, Y. H.; Kwong, C. Y.; Cheah, K. W.; Rao, T. K. G.; Chan, W. K.; Lui, H. F.; Surya, C. Photoluminescence and Electron Paramagnetic Resonance of ZnO Tetrapod Structures. Adv. Funct. Mater. 2004, 14, 856-864.

34. Liu, C.-C.; Liu, S.-H.; Tien, K.-C.; Hsu, M.-H.; Chang, H.-W.; Chang, C.-K.; Yang, C.-J.; Wu, C.-C. Microcavity TopEmitting Organic Light-Emitting Devices Integrated with Diffusers for Simultaneous Enhancement of Efficiencies and Viewing Characteristics. Appl. Phys. Lett. 2009, 94, 103302.

35. Lee, J.; Hofmann, S.; Furno, M.; Thomschke, M.; Kim, Y. H.; Lüssem, B.; Leo, K. Influence of Organic Capping Layers on the Performance of Transparent Organic Light-Emitting Diodes. Opt. Lett. 2011, 36, 1443-145. 